\title{
How tidal erosion has shaped the relation between globular cluster specific frequency and galaxy luminosity
}

\author{
S. Mieske ${ }^{1}$, A. H. W. Küpper ${ }^{2,3}$, and M. Brockamp ${ }^{4}$ \\ 1 European Southern Observatory, 3107 Alonso de Córdova, Vitacura, Santiago, Chile \\ e-mail: smieske@eso.org \\ 2 Department of Astronomy, Columbia University, 550 West 120th street, New York NY 10027, USA \\ 3 Hubble Fellow \\ ${ }^{4}$ Helmholtz-Institut für Strahlen- und Kernphysik, Universität Bonn, Nussallee 14-16, 53121 Bonn, Germany
}

Received 16 April 2014 / Accepted 29 April 2014

\begin{abstract}
Aims. We quantify to what extent tidal erosion of globular clusters (GCs) has contributed to the observed U-shaped relation between GC specific frequencies $S_{N}$ and host galaxy luminosity $M_{V}$.

Methods. We used our MUESLI code to calculate GC survival rates for typical early-type galaxy potentials covering a wide range of observed galaxy properties. We do this for isotropic and radially anisotropic GC velocity distributions.

Results. We find that the calculated GC survival fraction, $f_{\mathrm{s}}$, depends linearly on the logarithm of the 3D mass density, $\rho_{3 \mathrm{D}}$, within the galaxy's half-light radius, with $f_{\mathrm{s}} \propto \rho_{3 \mathrm{D}}^{-0.17}$. For a given galaxy, survival rates are lower for radially anisotropic configurations than for the isotropic GC cases. We apply these relations to a literature sample of 219 early-type galaxies from Harris et al. (2013, ApJ, $772,82)$ in the range $M_{V}=[-24.5:-15.5]$ mag. The expected GC survival fraction ranges from $50 \%$ for the most massive galaxies with the largest radii to $10 \%$ for the most compact galaxies. We find that intermediate luminosity galaxies $M_{V}=[-20.5:-17.5]$ mag have the strongest expected GC erosion. Within the literature sample considered, the predicted GC survival fraction therefore defines a U-shaped relation with $M_{V}$, similar to the relation between specific frequency $S_{N}$ and $M_{V}$. As a consequence, the U-shape of $S_{N}$ vs. $M_{V}$ gets erased almost entirely when correcting the $S_{N}$ values for the effect of GC erosion.

Conclusions. Tidal erosion is an important contributor to the U-shaped relation between GC specific frequency and host galaxy luminosity. It must be taken into account when inferring primordial star cluster formation efficiencies from observations of GC systems in the nearby universe.
\end{abstract}

Key words. stars: kinematics and dynamics - galaxies: star clusters: general - galaxies: stellar content - galaxies: evolution

\section{Introduction}

Globular clusters (GCs) are the fossil remnants of star formation in the very early universe. Interestingly, it has been found that the observed specific frequency $S_{N}=N_{\mathrm{GC}} \cdot 10^{0.4\left(M_{V}+15\right)}$ of GCs in galaxies systematically changes as a function of host galaxy luminosity $M_{V}$ (e.g. Forbes et al. 2005; Brodie \& Strader 2006; Peng et al. 2008; Spitler et al. 2008; Georgiev et al. 2010; Harris et al. 2013). It is high for very luminous and faint galaxies and comparably low for intermediate luminosity galaxies. In Fig. 1 this is shown for the 219 early-type galaxies (E/SO \& dE/SO) from the recent compilation of Harris et al. (2013) for which estimates of $S_{N}$ and dynamical mass exist. The luminosity range $M_{V}=[-24.5:-15.5]$ mag covered by the sample corresponds to a dynamical mass range $M_{\text {dyn }} \sim 10^{12.5}$ to $M_{\text {dyn }} \sim 10^{8.5} M_{\odot}$.

Discussion of this U-shape has focussed on systematic variations of field star vs. star cluster formation efficiencies across galaxies. Indeed, the observed number of GCs per total galaxy halo mass (i.e. including the full dark matter halo) has a significantly smaller variation than the specific frequency normalised by galaxy luminosity (Spitler et al. 2008; Georgiev et al. 2010; Harris et al. 2013; Hudson et al. 2014). As a result, one commonly cited explanation for the U-shape is that the GC formation efficiency is close to uniform per unit total halo mass, while field star formation was partially suppressed in very massive haloes and in dwarf galaxies (Dekel \& Birnboim 2006). The latter is supported by a similar U-shape of dynamical mass-to-light ratios for galaxies (Peng et al. 2008).

However, both Peng et al. (2008) and Georgiev et al. (2010) stress that the specific frequencies of GCs observed in the nearby universe are a combination of (at least) two effects: the ratio of star-cluster and field-star formation efficiencies, and the GC destruction rate. Indeed, Murali \& Weinberg (1997) and Vesperini (2000) have already suggested that the tidal destruction of GCs is an important contributor to observed variations in specific frequency with host-galaxy properties. Several more studies have since investigated the destruction of GCs in an external tidal field (e.g. Baumgardt 1998; Fall \& Zhang 2001; Vesperini et al. 2003; Sanchez-Janssen et al. 2012; Smith et al. 2013). However, a quantitative evaluation of GC erosion as a function of galaxy properties has been difficult up to now because of the numerical challenges associated with a full treatment of the problem. There are several internal (stellar evolution, two-body relaxation) and external (tidal forces, dynamical friction) processes acting simultaneously on GCs, with efficiencies depending strongly on the respective clusters' masses and orbits.

In Brockamp et al. (2014), the tidal erosion of GC systems in elliptical galaxies is systematically investigated with $N$-body simulations for a wide range of host galaxy masses and radii, as well as different initial GC velocity distributions. One of the 

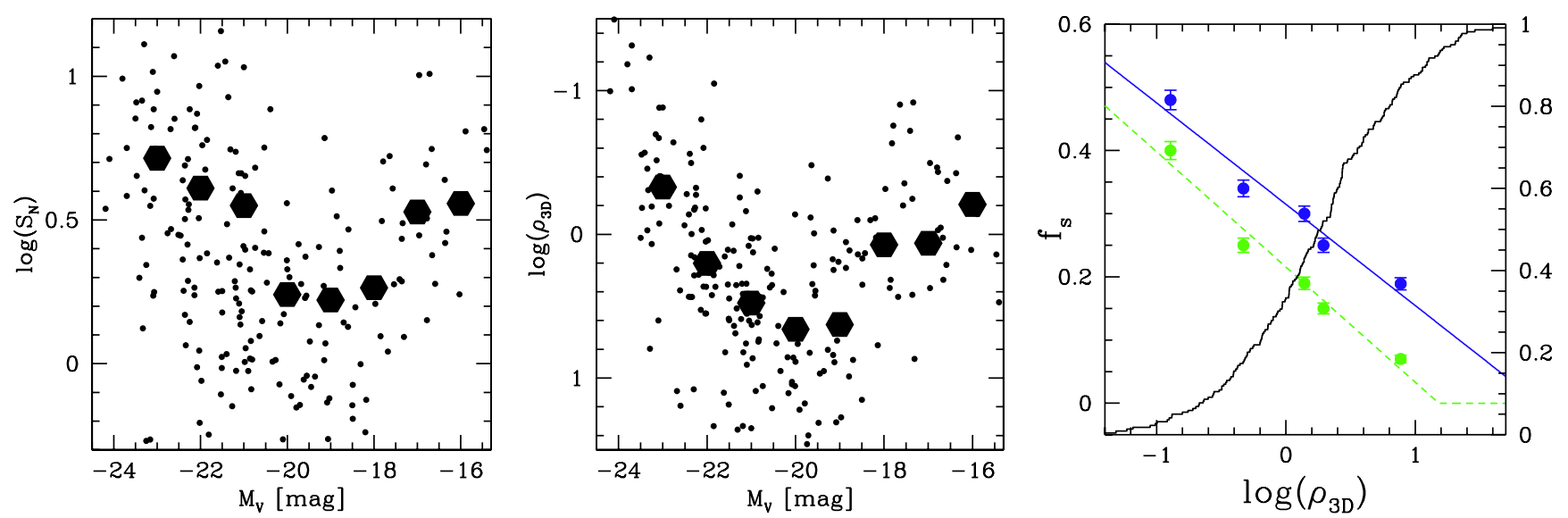

Fig. 1. GC specific frequencies and galaxy mass densities define similar U-shape relations with $M_{V}$. Left: $\operatorname{logarithm}$ of the specific frequency, $S_{N}$, of GCs plotted vs. the host galaxy luminosity, $M_{V}$. The sample is taken from the compilation of Harris et al. (2013), but restricted to early-type galaxies $(\mathrm{E} / \mathrm{SO}, \mathrm{dE} / \mathrm{SO})$ for which dynamical mass estimates exist. We also indicate the mean $S_{N}$ in bins of one magnitude (thick hexagons). Middle: logarithm of the projected 3D mass density within the half-light radius, $\rho_{3 \mathrm{D}}$, plotted vs. $M_{V}$ for the same sample as in the left panel. The units of the mass density are $M_{\odot} \mathrm{pc}^{-3}$. Right: survival fraction, $f_{\mathrm{S}}$, of GCs with masses $>10^{5} M_{\odot}$ after 10 Gyr of evolution plotted vs. the logarithm of the projected 3D mass density, $\rho_{3 \mathrm{D}}$, within the half-light radius of their host galaxy. The data are based on the simulations presented in Brockamp et al. (2014). The blue filled circles (fit by a solid blue line) correspond to the assumption of an isotropic GC velocity distribution, whereas the green filled circles (fit by a dashed green line) correspond to a modestly radially biased GC configuration. From the high to low survival fraction, the data correspond to the following template simulations: NGC 4889, VCC 1073, IC 1459, NGC 4494, and NGC 4564 (see also the summary in Table 1). The solid black curve shows the cumulative $\rho_{3 \mathrm{D}}$ distribution of the sample of early type galaxies considered in the left and middle panels.

main findings of this study is that, after $10 \mathrm{Gyr}$ of dynamical evolution, very compact galaxies like M 32 have GC survival fractions of only a few percent, while very extended giant elliptical galaxies have survival fractions of up to $50 \%$. Futhermore, it is found that GC number density profiles are centrally flattened in less than a Hubble time, naturally explaining the observed cored GC distributions that are generally more extended than the galaxy star light (e.g. Peng et al. 2011; Forbes et al. 2012).

The aim of the present letter is to quantify the importance of the tidal erosion of GC systems in shaping the observed relation between the specific frequency of GCs, $S_{N}$, and a galaxy's absolute luminosity, $M_{V}$. Based on the results of Brockamp et al. (2014), we compare the predicted survival fraction of GCs in early-type galaxies to the observed specific frequency of GCs using the recent compilation of Harris et al. (2013) and discuss our findings.

\section{Erosion of GC systems}

In the middle panel of Fig. 1 we plot the logarithm of the 3D dynamical mass density within the half-light radius, $\rho_{3 \mathrm{D}}$, versus the absolute luminosity, $M_{V}$, for the same compilation from Harris et al. (2013) as in the left-hand panel. A U-shape trend similar to the left-hand panel is seen. As shown in the following, the GC survival fraction depends linearly on $\log \left(\rho_{3 \mathrm{D}}\right)$, implying that part of the U-shape in $S_{N}$ vs. $M_{V}$ arises from GC erosion.

\subsection{A simple formula derived from Brockamp et al. (2014)}

In Brockamp et al. (2014), we presented the versatile MUESLI code for investigating the erosion of GC systems in elliptical galaxies. MUESLI follows the orbits of individual clusters in galaxy potentials and applies both internal and external dissolution processes to them, yielding GC destruction rates as a function of host galaxy parameters. In a first application, we studied spherical galaxies with different masses, sizes, central super-massive black holes, density profiles, and velocity distributions. We found that the erosion efficiency strongly depends on the compactness of the galaxy and the anisotropy of its velocity distribution.

For the present study, we consider Sérsic galaxy models that have concentration parameters $n=4$ (and $n=$ 2.99 for VCC 1073) with both initially isotropic and initially radial-biased velocity distributions for the GC orbits. The anisotropic velocity distribution follows Osipkov-Merritt models (Osipkov 1979; Merritt 1985) with a characteristic anisotropy radius that equals the $3 \mathrm{D}$ half mass radius $(\approx 1.35$ times the projected half-light radius, $r_{\mathrm{h}}$ ). This choice of parameter yields a moderately anisotropic velocity distribution, with the central parts still isotropic and the outer parts more radially biased (see e.g. Fig. A1 in Brockamp et al. 2014). At the end of the simulations after $10 \mathrm{Gyr}$, the central orbital distribution has become tangentially biased due to the higher destruction of GCs on radial orbits.

We assume that the galaxies have no central SMBH, because we found in Brockamp et al. (2014) that these have very little effect on GC erosion in general. The total number of GCs at the start of the simulations was 20000 , sampled from a power-law mass function reaching down to $10^{4} M_{\odot}$ (see Brockamp et al. for details). To establish comparability between our simulations and the typical completeness limit for observations of GC systems (e.g. Harris et al. 2013), we restricted ourselves to simulated clusters with masses above $10^{5} M_{\odot}$. To cover the full range of 3D mass densities from Harris et al. (2013), we made two new simulations (representing NGC 4564 and VCC 1073) following the setup described in Brockamp et al. (2014). A summary of the simulation results for the five galaxies can be found in Table 1.

The results can be expressed as a relation between GC survival fraction and the logarithm of the 3D dynamical mass density within the half-light radius. In Fig. 1 (right panel), we plot the GC survival fraction, $f_{\mathrm{s}}$, versus the logarithm of the $3 \mathrm{D}$ mass density within the half-light radius, $\rho_{3 \mathrm{D}} \equiv M_{\mathrm{dyn}} /\left(r_{\mathrm{h}}^{3}\right)$. It is apparent that radial anisotropy leads to a higher GC destruction rate as more clusters orbit through the denser central parts of the 

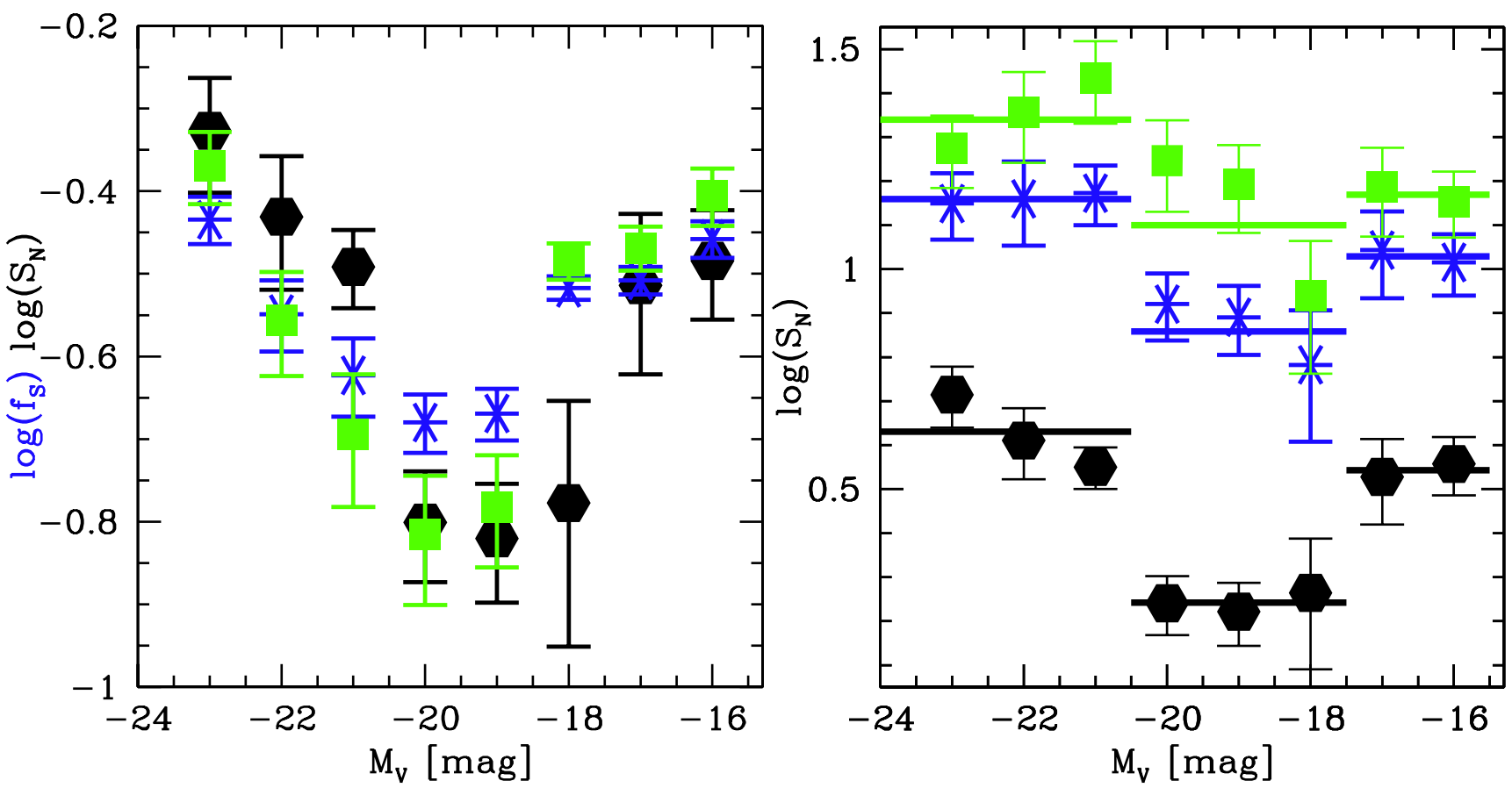

Fig. 2. How tidal erosion changes the relation between GC specific frequency $S_{N}$ and galaxy luminosity $M_{V}$. Left: we compare the observed GC specific frequencies, $S_{N}$ (offset by a constant factor), with the predicted GC survival fractions, $f_{\mathrm{S}}$, for the same set of galaxies from Fig. 1, using the predictions from Eqs. (1) and (2). Large symbols indicate means within 1 mag bins. The observed $S_{N}$ are indicated in (black) hexagons, survival fractions in (blue) asterisks for isotropic orbits and in (green) squares for radially anisotropic orbits. Right: predicted primordial specific frequencies are obtained by dividing the observed specific frequencies by the survival fractions of the left panel. Colours and symbols as in the left panel. Black hexagons are again the observed present-day specific frequencies. The U-shape is attenuated for the isotropic case and is almost erased for the anisotropic case, see text for details. Horizontal thick lines denote the average $S_{N}$ values within the three luminosity ranges considered, $M_{V}=[-24.5:-20.5]$ (high luminosity), $M_{V}=[-20.5:-17.5]$ (intermediate luminosity), $M_{V}=[-17.5:-15.5]$ (low luminosity).

Table 1. Main parameters of the five GC-system erosion simulations shown in Fig. 1.

\begin{tabular}{lllrlll}
\hline \hline Galaxy & $r_{\mathrm{h}}$ & $M_{\text {dyn }}$ & $\log \left(\rho_{3 \mathrm{D}}\right)$ & $f_{\mathrm{s}, \text { iso }}$ & $f_{\text {s,aniso }}$ & $M_{V}$ \\
\hline NGC 4564 & 1.78 & 43.5 & 0.89 & 0.19 & 0.07 & -20.0 \\
NGC 4494 & 3.72 & 100 & 0.29 & 0.25 & 0.15 & -22.0 \\
IC 1459 & 6.0 & 300 & 0.14 & 0.30 & 0.19 & -22.7 \\
VCC 1073 & 2.0 & 3.75 & -0.33 & 0.34 & 0.25 & -17.5 \\
NGC 4889 & 25.0 & 2000 & -0.89 & 0.48 & 0.40 & -24.2 \\
\hline
\end{tabular}

Notes. Half-light radius, $r_{\mathrm{h}}$, is given in kpc, dynamical galaxy mass, $M_{\mathrm{dyn}}$, is given in units of $10^{9} M_{\odot}$. The survival fraction, $f_{\mathrm{s}}$, adopted here is the ratio of the number of GCs with mass $M>10^{5} M_{\odot}$ after $10 \mathrm{Gyr}$ to the number of GCs with mass $M>10^{5} M_{\odot}$ at the start of the simulation. We show the results both for the isotropic and the radially anisotropic cases.

galaxy. In Fig. 1 we indicate a linear fit to the five data points for both cases of isotropy and radial anisotropy. These linear fits are given by

$$
\begin{aligned}
& f_{\mathrm{s}, \text { iso }}=-0.160 \cdot \log _{10}\left(\rho_{3 \mathrm{D}}\right)+0.315 \\
& f_{\mathrm{s} \text {,aniso }}=-0.182 \cdot \log _{10}\left(\rho_{3 \mathrm{D}}\right)+0.216 .
\end{aligned}
$$

We note that we do not include our simulation for M 32 at very high density, since it is in a density regime where the GC survival fraction is already "saturated" at 0 . The histogram in Fig. 1 shows the cumulative distribution of $3 \mathrm{D}$ mass densities, $\rho_{3 \mathrm{D}}$, for the literature sample that defines the $S_{N}$ vs. $M_{V}$ relation in Figs. 1 and 2. The range of 3D density of the simulated GC systems covers the range of the literature sample.

\subsection{Application to the sample of Harris et al. (2013)}

In the left-hand panel of Fig. 2, we have calculated the GC survival fraction for the early-type galaxies in the sample of Fig. 1, using the observed galaxy mass densities and the relation between survival fraction and mass density from Eqs. (1) and (2). For the sake of clarity we focus on average values within 1 magnitude bins and omit individual data points. We indicate the logarithm of the expected survival fraction for an isotropic velocity distribution (Eq. (1)) and a radially biased velocity distribution (Eq. (2)). For comparison we over-plot the present-day specific frequencies (same data points as in the left panel of Fig. 1), shifted by a constant offset in log-space.

The most striking feature of Fig. 2 is that the expected survival fraction defines a U-shape that is very similar to the observed $S_{N}$ vs. $M_{V}$ data points. For the case of isotropic GC velocity distributions, the amplitude of variation in the predicted survival fractions is about a factor of two lower than the observed variation of specific frequencies: the ratio between the maximum and minimum survival rates within the eight magnitude bins is 1.8. For comparison, the ratio between maximum and minimum specific frequency $S_{N}$ within the same set of eight magnitude bins is 3.1 (5.2 to 1.7). Interestingly, for the case of radial anisotropy, the amplitude of the predicted U-shape relation matches the observations well. For this case, the ratio between the maximum and minimum survival rate within the eight magnitude bins is 2.8 , matching the observed amplitude variation to within $10 \%$.

In the right-hand panel we show the "primordial" specific frequencies of the same galaxies, obtained by dividing the observed $S_{N}$ values by the survival fractions. We consider 
Table 2. KS-test probabilities that the $S_{N}$ distribution within the three subsamples (high, intermediate [=int.], low luminosity) have the same parent distribution.

\begin{tabular}{llll}
\hline \hline KS probabilities & High vs. int & High vs. low & Int. vs low \\
\hline Present-day & $5 \times 10^{-6}$ & 0.70 & $1.5 \times 10^{-3}$ \\
Prim. iso & $1 \times 10^{-4}$ & 0.30 & 0.15 \\
Prim. aniso & 0.10 & 0.53 & 0.44 \\
\hline
\end{tabular}

Notes. See also the right panel of Fig. 2. We show these probabilities for the observed $S_{N}$, and for the primordial $S_{N}$ before erosion, both for isotropic and radially anisotropic GC velocity distributions.

the three subsamples $M_{V}=[-24.5:-20.5]$ (high luminosity), $M_{V}=$ [-20.5:-17.5] (intermediate luminosity), $M_{V}=$ [-17.5:-15.5] (low luminosity). For the case of an isotropic GC velocity distribution, the predicted primordial specific frequencies are 14.4, 7.2, 10.7 in those three ranges. For radial anisotropy, these predictions are 22.9, 14.0, 14.8 .

In Table 2 we show the results of a Kolmogorov-Smirnov (KS) test comparing the $S_{N}$ distribution of the high, intermediate, and low-luminosity samples. We consider the following three cases: observed $S_{N}$, inferred primordial $S_{N}$ for both isotropic and anisotropic GC velocity distributions. The characteristic U-shape in the observed $S_{N}$ vs. $M_{V}$ plane is clearly reflected in the KS probabilities: the intermediate-luminosity sample is grossly inconsistent with both high- and low-luminosity galaxies, while the other two agree well. The situation gradually changes for the primordial $S_{N}$ with isotropic GC orbits, and the disagreement between the subsamples disappears for primordial $S_{N}$ based on anisotropic GC orbits. The comparison between high- and intermediate-luminosity samples for the latter case gives a comparably high $(10 \%)$ probability that both have the same parent distribution.

\section{Summary and conclusions}

Applying the simulation results of GC erosion in elliptical galaxies from Brockamp et al. (2014), we calculated the survival rates of GCs in early type galaxies, using a sample of 219 early type galaxies in the $M_{V}=[-24.5:-15.5] \mathrm{mag}$.

We show that the simulations of GC system erosion in Brockamp et al. (2014) are approximated well by a linear relation between GC survival fraction and the logarithm of the $3 \mathrm{D}$ dynamical mass density within the galaxy's half-light radius $\left(f_{\mathrm{s}} \propto \rho_{3 \mathrm{D}}^{-0.17}\right.$.). For a given galaxy, survival rates are lower for radially anisotropic $\mathrm{GC}$ velocity distributions than for isotropic ones. Within the considered literature sample from Harris et al. (2013), the expected GC survival fraction ranges from 50\% for the most massive galaxies with the largest radii to $10 \%$ for the most compact galaxies.

Within the considered literature sample, the predicted GC survival fraction defines a similar U-shaped relation with $M_{V}$ than the specific frequency $S_{N}$. In particular, the peak-tovalley of the survival fraction when considering moderate radial anisotropy agrees to within $10 \%$ with the peak-to-valley of the $S_{N}$. Higher degrees of primordial anisotropy may reconcile the results completely.
As a consequence, the U-shape of $S_{N}$ vs. $M_{V}$ becomes almost flat when correcting the $S_{N}$ values for the effect of GC erosion for radially anisotropic orbits. A KS test shows no significant difference in corrected $S_{N}$ distribution between high-, intermediate- and low-luminosity galaxies. The original U-shape is notably attenuated but still preserved for the assumption of an isotropic GC velocity distribution.

We therefore find that GC erosion is a major contributor to the U-shaped relation between present-day GC specific frequency, $S_{N}$, and host galaxy luminosity, $M_{V}$. It must be taken into account when inferring primordial star cluster formation efficiencies from observations of GC systems in the nearby universe, and may thus change our picture of how field star and star cluster formation were coupled in the early universe.

We conclude with a note of caution. It is unlikely that tidal erosion alone is responsible for the entire variation in $S_{\mathrm{N}}$ vs. $M_{V}$ we observe today. For example, our simulations (Brockamp et al. 2014) do not explicitly include the hierarchical nature of structure formation in the universe. Current GC systems of most galaxies are probably compounds of smaller GC systems acquired through mergers (e.g. van Dokkum et al. 2008). Blue and red GCs have different spatial distributions (e.g. Brodie \& Strader 2006), an effect not yet considered in our simulations. Furthermore, host-galaxy potentials should be evolving with time. A detailed inclusion of such effects into simulations of GC system dynamical evolution is thus a promising prospect.

Acknowledgements. The authors would like to thank the anonymous referee for valuable comments, and J.P. Ostriker for interesting discussions. We thank Michael Marks for interesting discussions and Holger Baumgardt for technical support. S.M. acknowledges support from the ESO Director General Discretionary Fund and the kind hospitality of the Columbia University Astronomy Department. A.H.W.K. acknowledges support through Hubble Fellowship grant HST-HF-51323.01-A awarded by the Space Telescope Science Institute, which is operated by the Association of Universities for Research in Astronomy, Inc., for NASA, under contract NAS 5-26555. M.B. acknowledges support through DFG grant KR 1635/39-1.

\section{References}

Baumgardt, H. 1998, A\&A, 330, 480

Brockamp, M., Kuepper, A. H. W., Thies, I., Baumgardt, H., \& Kroupa, P. 2014, MNRAS, 441, 150

Brodie, J. P., \& Strader, J. 2006, ARA\&A, 44, 193

Dekel, A., \& Birnboim, Y. 2006, MNRAS, 368, 2

Fall, S. M., \& Zhang, Q. 2001, ApJ, 561, 751

Forbes, D. A. 2005, ApJ, 635, L137

Forbes, D. A., Ponman, T., \& O'Sullivan, E. 2012, MNRAS, 425, 66

Georgiev, I. Y., Puzia, T. H., Goudfrooij, P., \& Hilker, M. 2010, MNRAS, 406, 1967

Harris, W. E., Harris, G. L. H., \& Alessi, M. 2013, ApJ, 772, 82

Hudson, M. J., Harris, G. L., \& Harris, W. E. 2014, ApJ, submitted [arXiv: 1404.1920]

Merritt, D. 1985, AJ, 90, 1027

Murali, C., \& Weinberg, M. D. 1997, MNRAS, 288, 767

Osipkov, L. P. 1979, Sov. Astron. Lett., 5, 42

Peng, E. W., Jordán, A., Côté, P., et al. 2008, ApJ, 681, 197

Peng, E. W., Ferguson, H. C., Goudfrooij, P., et al. 2011, ApJ, 730, 23

Sánchez-Janssen, R., \& Aguerri, J. A. L. 2012, MNRAS, 424, 2614

Smith, R., Sánchez-Janssen, R., Fellhauer, M., et al. 2013, MNRAS, 429, 1066

Spitler, L. R., Forbes, D. A., Strader, J., Brodie, J. P., \& Gallagher, J. S. 2008, MNRAS, 385, 361

van Dokkum, P. G., Franx, M., Kriek, M., et al. 2008, ApJ, 677, L5

Vesperini, E. 2000, MNRAS, 318, 841

Vesperini, E., Zepf, S. E., Kundu, A., \& Ashman, K. M. 2003, ApJ, 593, 760 